\title{
DISCONNECTED REGIONS OF STABLE CIRCULAR ORBITS IN PRESENCE OF MASSIVE SCALAR FIELD
}

\author{
O.S. Stashko ${ }^{1}$, V.I. Zhdanov ${ }^{2}$ \\ 1 Taras Shevchenko National University of Kyiv, Physical Faculty, \\ Kiev, Ukraine, alexander.stashko@gmail.com \\ 2 Taras Shevchenko National University of Kyiv, Astronomical observatory, \\ Kiev, Ukraine, ValeryZhdanov@gmail.com
}

ABSTRACT. We study circular orbits around a static spherically symmetric configuration of General Relativity in presence of a nonlinear massive scalar field (SF). The problem deals with a family of solutions to Einstein - SF equations with the SF potentials that are negative for some SF values in order to consider black hole configurations. We show that, for some parameters of the family, there can exist two disconnected regions of stable circular orbits around the configuration. Such regions can exist both for black hole and for naked singularities.

Keywords: classical black holes, naked singularities, scalar fields, accretion disks.

\section{Introduction}

Scalar field (SF) is often used in the General Relativity to study models of the dynamical dark energy (Novosyadlyi et al. 2013), so it is natural to look for the SF effects in astrophysical objects. Here we look for such effects in stable circular orbits (SCO) distributions around spherically symmetric configuration. It is well known that SCO form a connected structure in Schwarzschild and Kerr black hole space-times of General Relativity. It was shown by Chowdhury et al. (2012) that, in presence of the linear massless scalar field, there can exist disconnected regions of SCO separated by a ring of unstable orbits. Examples of gaps in the SCO distribution have been found by Stuchlík \& Schee (2010); Pugliese et al. (2013, 2017); Vieira et al. (2014); Boshkayev et al. (2016) in presence of naked singularities (NS). We wonder, is NS always necessary for the existence of such a discontinuous SCO structure?

The aim of this paper is to present examples with a non-trivial self-interaction potential $V(\phi)$ of the massive scalar field $\phi$ showing that the disconnected SCO structure can exist in the black hole $(\mathrm{BH})$ space-time as well. We study test-body circular orbits in static spherically symmetric asymptotically flat space-times of General Relativity in presence a non-linear SF minimally coupled with gravity. Note that our black hole solutions discussed below involve potentials that are negative in some regions, so they do not contradict to the no-scalar-hair theorem (Bekenstein 1998).

We shall use a well known method (see, e.g., Bronnikov 2001, Bronnikov \& Shikin 2002, Azreg-Aïnou 2010) of generation of special spherically symmetric solutions to these equations along with corresponding potentials. Investigation shows the occurrence of the disconnected SCO distribution, both in case of $\mathrm{BH}$ and/or NS, for certain domain of the family parameters. At the same time, the other choice of the parameters can lead to the absence of discontinuities in the SCO distribution. Here we present main results of a numerical investigation; more detailed presentation can be found in our preprint (Stashko \& Zhdanov 2017) and will be published elsewhere.

\section{Basic relations}

The metric of a general spherically symmetric spacetime in static region can be written as

$$
d s^{2}=A(x) d t^{2}-\frac{d x^{2}}{A(x)}-r^{2}(x) d O^{2},
$$

and we say that $x_{0}$ is a point of a center if $r\left(x_{0}\right)=0$ and $r(x)>0$ for $x>x_{0}$. The center $x_{0}$ appears to be a simple root of $r(x)$ (Bronnikov 2001, Bronnikov \& Shikin 2002, Azreg-Aïnou 2010).

The Einstein equations in presence of a selfinteracting minimally coupled scalar field $\phi$ can be derived from the action functional

$$
S=S_{G R}+\int d^{4} x \sqrt{|g|}\left[g^{\mu \nu} \phi_{, \mu} \phi_{, \nu}-2 V(\phi)\right],
$$

where $S_{G R}$ is the standard gravitational action of the General Relativity $(c=8 \pi G=1)$, and $V(\phi)$ is a selfinteraction potential to be specified below. The joint system of equations for $A, r, \phi$ under the conditions of spherical symmetry and asymptotic flatness is reduced to a system of equations (Azreg-Aïnou 2010), 
which represent a general solution in an implicit form in terms of arbitrary $r(x)$. Thus, we use the "inverse" method (Bronnikov 2001, Bronnikov \& Shikin 2002) to generate families of special solutions: we fix $r(x)$, and look for $A(x), \phi(x)$ and $V(\phi)$. This problem is solved uniquely under the conditions of the asymptotic flatness, where $V(\phi)$ is defined parametrically. In particular,

$$
A(x)=r^{2}(x) \int_{x}^{\infty} \frac{2 x^{\prime}-C}{r^{4}\left(x^{\prime}\right)} d x^{\prime},
$$

where for the integration constant we have $C=6 \mathrm{~m}$, and $m>0$ is the mass of the whole configuration.

Further we make a particular choice

$$
r(x)=x\left[1-\left(\frac{x_{0}}{x}\right)^{N} e^{-\mu\left(x-x_{0}\right)}\right],
$$

where $x_{0} \geq 0, N>1$, and $\mu>0$ is related to the scalaron mass $\mu / 2$. The latter follows from the estimate (Stashko \& Zhdanov 2017)

$$
V(\phi) \sim \frac{\mu^{2}}{8} \phi^{2}
$$

for small $\phi$ (and any fixed $N$ ) on account of asymptotic relations for $x \rightarrow \infty$.

We use the general results of Azreg-Aïnou (2010) showing that (a) if $x_{0} \geq 3 \mathrm{~m}$, then $A(x)>0, x>x_{0}$, and we have a naked singularity at $x \rightarrow x_{0}$; (b) if $x_{0}<3 m$, then we have a BH solution: there exist a point $x_{h}>x_{0}$ (the horizon) such that $A\left(x_{h}\right)=0$ and $A(x)>0$ for $x>x_{h}$. For numerical estimates we choose further the units of length so as to have $C=1$. The SF potentials that correspond to (4) for different values of the parameters are shown on Fig.1. For $x_{0}<0.5$ (BH case) they have a form of the Mexican hat and they are not positive everywhere; for $x_{0} \geq 0.5$ (NS case) the potentials are not bounded from below.

\section{Regions of SCO}

We are interested in time-like geodesics of the static region, where $A>0, r>0$. We shall concentrate on the case shown in Fig.2: here $X_{r}, r=1,2,3$ represent limiting radii of different regions of the stable circular orbits. Namely, the radial coordinates $x: X_{3}<x<\infty$ correspond to the infinite outer ring of circular orbits, and $x: X_{1}<x<X_{2}$ correspond to the inner ring (if it exists), which is separated off the outer region by a prohibited area $X_{2}<x<X_{3}$, where either there is no circular orbits or they are unstable. In case of the spherically symmetric space-time metric (1) the investigation of geodesics is reduced to the equations of one-dimensional classical particle motion in a field with effective potential $U_{\text {eff }}(x, L)=L^{2} U_{1}(x)+U_{2}(x)$, where

$$
U_{1}(x)=\frac{A(x)}{r^{2}(x)}, \quad U_{2}(x)=A(x) .
$$

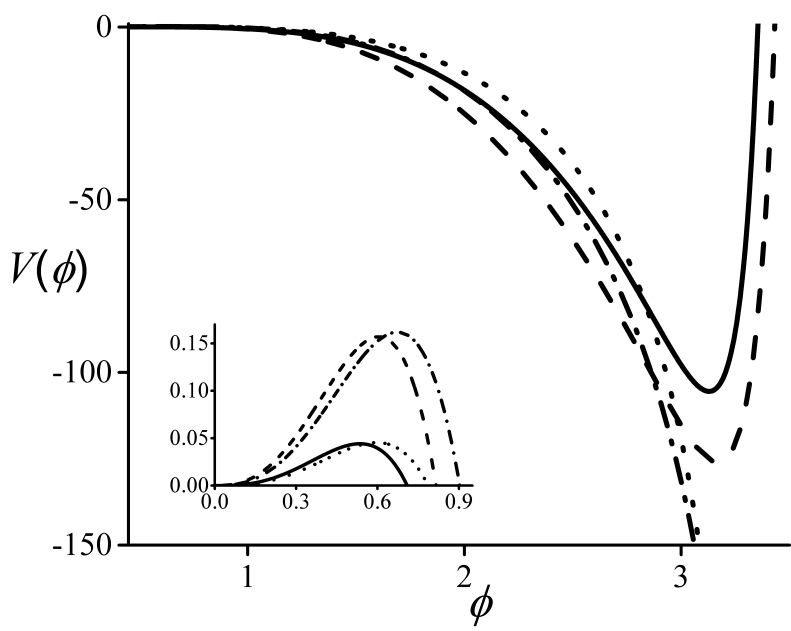

Figure 1: Scalar field potentials in cases of $\mathrm{BH}\left(x_{0}=\right.$ $0.45, \mu=0: N=4-$ solid, $N=5-$ dashed) and NS $\left(x_{0}=0.55, \mu=0: N=4\right.$ - dotted, $N=5$ - dashdot). Smaller panel shows details of the graphs near the origin $\phi=0$. which cannot be seen on the scale of the larger panel. We note that the potential graph of the intermediate case $x_{0}=0.5$ (not shown here) is completely similar to the case of $x_{0}>0.5$.

At the points of minima of $U_{\text {eff }}$ we have $U_{\text {eff }}^{\prime}=$ $0, U_{e f f}^{\prime \prime}>0$ yielding

$$
L^{2}=F(x), \quad F^{\prime}(x)>0,
$$

where

$$
F(x)=-U_{2}^{\prime} / U_{1}^{\prime}
$$

The roots of the equation (7) in the region where $F^{\prime}(x)>0, F(x)>0$ correspond to SCO with different radii. The double roots of (7) yield double minima of $U_{\text {eff }}$ with fixed $L$. Simple analysis on account of the explicit form of $r(x)$ (see Stashko \& Zhdanov 2017) shows that in order to have non-connected regions of SCO one must have a positive maximum of $F(x)$. Then the limiting radii of the SCO regions can be found either as roots of the second derivative $F^{\prime \prime}(x)$, of as roots of the function $F(x)$ itself. Using the equation (3) one can find an explicit form of $F(x)$ and its derivatives in terms of $r(x)$ so as to find these roots numerically.

The conditions that guarantee the existence of separated SCO regions can be violated at bifurcation points in the space of parameters $x_{0}, N, \mu$. These can be two types of the bifurcations:

(I) when the point of the maximum becomes negative

$$
F^{\prime}(X)=0, \quad F(X)=0, \quad F^{\prime \prime}(X)<0 ;
$$

(II) when the maximum of $F(x)$ disappears: it merges together with a point of minimum to yield an inflection point:

$$
F^{\prime}(X)=0, \quad F^{\prime \prime}(X)=0, \quad F(X)>0 .
$$




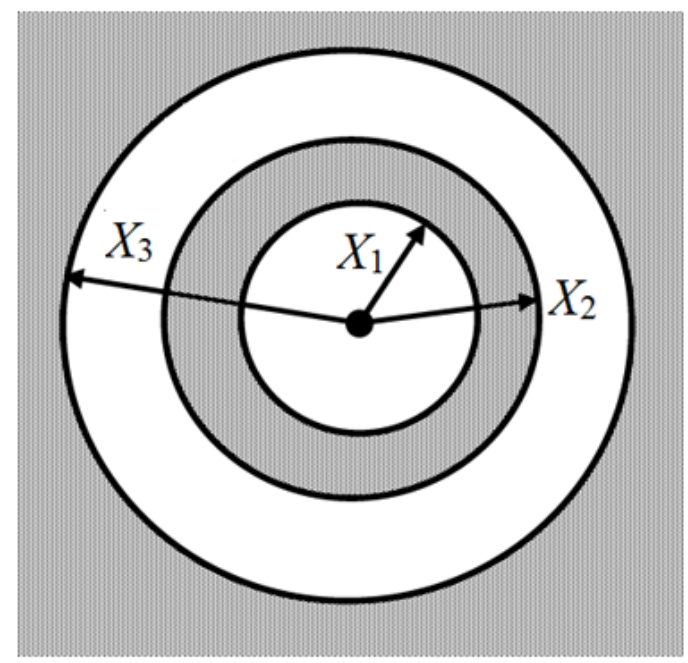

Figure 2: Shaded area: regions of stable orbits. White area: no stable orbits.

We performed this analysis in case of the family of solutions that correspond to the generating function (4). Fig.3 shows the resulting bifurcation cusp-like curves in the plane of parameters $N, x_{0}$ for several $\mu$. The region for $x_{0}<0.5$ (to the left of the vertical dotted straight line) describes the $\mathrm{BH}$ cases; this region contains the whole left branch of the "cusp" and a part of the lower branch. We note that for sufficiently small $x_{0}$ (sufficiently small SF effects) there is no disconnected SCO regions.

\section{Conclusions}

We studied special solutions of the joint system of Einstein equations and SF equations with various nonzero self-interaction potentials. The solutions describe isolated static spherically symmetric configurations with an asymptotically flat space-time and a positive total mass. These solutions deal with either NS in the center of the configuration, or with $\mathrm{BH}$. The family includes the Schwarzschild metric as limiting case $x_{0}=0$ and $V \equiv 0$. In the $\mathrm{BH}$ case, the scalar field potentials $V(\phi)$ have the form of the Mexican hat; they are bounded from below. The potentials are infinitely negative for some SF values in case of NS.

The main outcome of this paper is that separated (disconnected) ring-like structures of stable circular orbits do exist for some family parameters in the $\mathrm{BH}$ space-times. Analogous feature was first revealed by Chowdhury et al. (2012) in case of the massless linear scalar field dealing with NS at the center. The wellknown BH-no-hair theorem (Bekenstein 1998) does not prohibit the $\mathrm{BH}$ case, if the scalar field potential is not positive everywhere; this is just the case of our solutions. It is also important to note that occurrence of NS

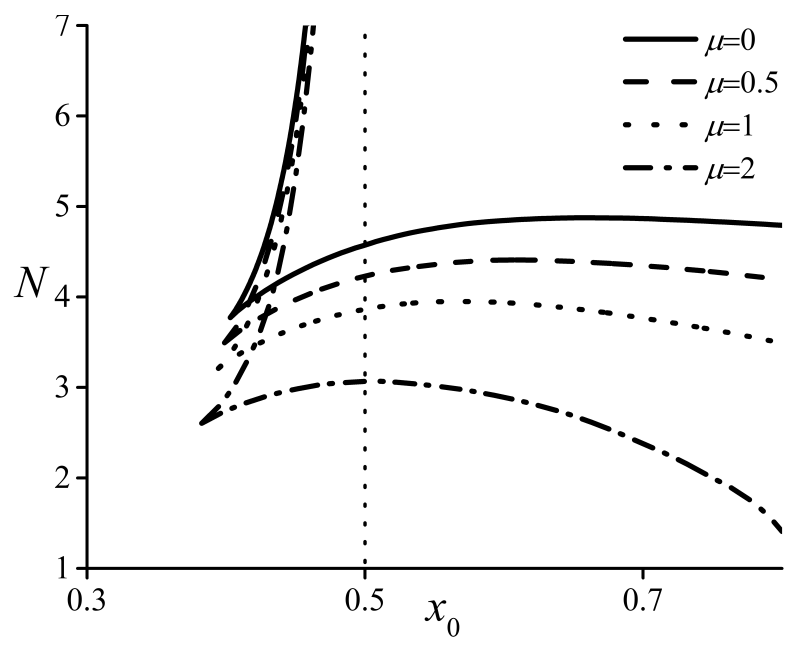

Figure 3: Cusp-like bifurcation curves limiting the regions of parameters where SCO form separate inner rings as in Fig.2. For any choice of the family parameters $N, x_{0}$ inside a cusp (to the right of the curves) the configuration has two different non-connected regions of SCO.

does not necessarily imply the existence of the discontinuous structures. There is no any universal situation with the scalar field as concerned the existence of the non-connected SCO regions, either in the $\mathrm{BH}$ or NS case.

Acknowledgements. This work has been supported in part by the Department of target training of Taras Shevchenko National University of Kyiv under National Academy of Sciences of Ukraine (project $6 \Phi$ ).

\section{References}

Azreg-Aïnou M., 2010, GRG, 42, 1427.

Bekenstein J.D.: 1998, Black Holes: Classical Properties, Thermodynamics and Heuristic Quantization. ArXiv:gr-qc/9808028.

Boshkayev K. et al.: 2016, PRD, 93, id.024024.

Bronnikov R.A.: 2001, PRD, 64, id.064013.

Bronnikov K.A., Shikin G.N.: 2002, Gravit. Cosmol., 8, 107.

Chowdhury A.N. et al.: 2012, PRD, 85, id.104031.

Novosyadlyi B., Pelykh V., Shtanov Yu., Zhuk A.: Dark energy and dark matter of the universe: in three volumes. Ed. V. Shulga, Vol. 1: Dark matter: Observational evidence and theoretical models (Kiev, Akademperiodyka, 2013) [arXiv:1502.04177].

Pugliese D. et al.: 2013, PRD, 88, id.024042.

Pugliese D. et al.: 2017, Eur. Phys. J. C, 77, id.206.

Stashko O.S., Zhdanov V.I.: 2017, arXiv:1702.02800v2.

Stuchlík Z., Schee J.: 2010, Class. Quant. Grav., 27, id. 215017.

Vieira R.S.S. et al.: 2014, PRD, 99, id.024035. 